\title{
A switched-capacitor PWM generator for LCD backlight brightness control
}

\author{
Sang-hyeok Yang ${ }^{1 \mathrm{a})}$, Kye-Shin Lee $^{2}$, Suki Kim ${ }^{1}$, \\ and Yong-Min Lee ${ }^{3}$ \\ ${ }^{1}$ Department of Electrical Engineering, Korea University, \\ Anam, Seongbuk-gu, Seoul,136-701, South Korea \\ ${ }^{2}$ Department of Electrical and Computer Engineering, The University of Akron, \\ Akron $\mathrm{OH}$ 44325, USA \\ ${ }^{3}$ Department of information display, Sun Moon University, \\ Asan-si, Chungnam, 336-708, South Korea \\ a)shyang@ulsi.korea.ac.kr
}

\begin{abstract}
In this paper, a pulse width modulation (PWM) generator based on a switched capacitor for LCD backlight brightness control is described. The proposed scheme uses a simple RC network along with a clocked-comparator which samples the output of the ambient light sensor, and generates a PWM signal corresponding to the light level. Furthermore, by using a switched-capacitor resistor with variable clock frequency, the RC time constant is programmable and less sensitive to process variations with respect to passive resistors. In addition, it is shown that the linearity of the proposed PWM generator can be enhanced by replacing the RC network resistor with a constant current source. The operation of the PWM generator is verified through circuit level simulations, and the prototype circuit is fabricated using CMOS 0.18 um technology.
\end{abstract}

Keywords: pulse width modulation, switched-capacitor, RC network, LCD backlight control

Classification: Integrated circuits

\section{References}

[1] J. Peng, et al., "High contrast LCD TV using active dynamic LED backlight," Symposium on Information Display (SID), pp. 1336-1338, 2005.

[2] K. Maeda, et al., "The system LCD with monolithic ambient-light sensor system," Symposium on Information Display (SID), pp. 356-358, 2005.

[3] Z. Lai and K. M. Smedley, "A general constant-frequency pulsewidth modulator and its applications," IEEE Trans. Circuits Syst. I, Fundam Theory Appl., vol. 45, no. 4, pp. 386-396, April 1998.

[4] B. J. Patella, et al., "High-frequency digital PWM controller IC for DCDC converters," IEEE Trans. Power Electron, vol. 18, no. 1, pp. 438-446, Jan. 2003. 


\section{Introduction}

An adaptive brightness control according to ambient light level has been used in LCD backlights for good visibility and power consumption reduction purpose $[1,2]$. Generally, PWM circuits have been used in LCD backlight drivers for controlling the brightness of the display panel. However, conventional PWM generators use a ramp signal generator in order to make a saw-tooth signal [3]. This scheme has a simple structure and shows good linearity between the input voltage and the output pulse width. However, the constant power consumption of the saw-tooth signal generator is a drawback for battery operated mobile applications. An alternative is the all-digital type PWM generator composed of logic circuits such as the inverter, counter and multiplexer [4]. Although digital logics consume relatively less power than the ramp signal generator, in order to convert the ambient light level into a digital code, an ADC is required. This can be problematic for mobile applications as well, since the area and power consumption increases proportional to the resolution of the ADC.

This paper presents a PWM generator composed of a simple RC network and a clocked-comparator for LCD backlight brightness control. Since the proposed scheme does not require a ramp signal generator or an ADC, the power consumption can be considerably reduced compared to conventional analog and all digital type PWM generators. Both simulation and measurement results show the validity of the proposed PWM generator, and the effectiveness of the linearity enhancement approach is verified as well.

\section{Proposed PWM generator}

Fig. 1 shows the proposed switched-capacitor PWM generator architecture which includes the ambient light sensor and readout circuitry, two switches, resistor-capacitor $(\mathrm{RC})$ network with capacitor $\mathrm{C}_{\mathrm{S}}$ and resistor $\mathrm{R}_{\text {eq }}$, and the comparator. In order to make the $\mathrm{RC}$ network less sensitive to process variations a switched-capacitor (SC) resistor is used instead of the passive resistor. The $\mathrm{SC}$ resistor consists of the sampling capacitor $\mathrm{C}_{\mathrm{eq}}$ and two switches that are controlled by two phase non-overlapping clocks $\Phi 1$ and $\Phi 2$ with sampling frequency of $f_{S}$. Furthermore, the resistance of the SC resistor, and the time constant of the RC network can be written as

$$
\begin{gathered}
R_{e q}=\frac{1}{f_{s} \cdot C_{e q}} \\
\tau=R_{e q} \cdot C_{s}=\frac{1}{f_{s}} \cdot \frac{C_{s}}{C_{e q}}
\end{gathered}
$$

As a result, the resistance can be changed by varying the control clock frequency $f_{S}$, and the time constant is simply given by the ratio between the two capacitors $\mathrm{C}_{\mathrm{S}}$ and $\mathrm{C}_{\mathrm{eq}}$.

The light sensor detects the ambient light level and converts it into a voltage signal. The readout circuitry amplifies the sensor output and adjusts the voltage level such that it can be processed in the following stages. The 
operation of the output pulse generator consists of two phases. In the sampling phase, when SW1 is on and SW2 is off, the voltage corresponding to the ambient light level is sampled in capacitor $\mathrm{C}_{\mathrm{S}}$. During the discharge phase, when SW2 is on and SW1 is off, the RC network (node $V_{X}$ ) is connected to the positive input of the comparator. Therefore, at the beginning of the discharge phase, in case there is a considerable amount of ambient light, the comparator output will be high. However, as the charge stored in $\mathrm{C}_{\mathrm{S}}$ is discharged through the resistor $R_{\text {eq }}$, the node voltage $V_{X}$ will decrease, and once $\mathrm{V}_{\mathrm{X}}$ reaches the threshold level $\mathrm{V}_{\mathrm{R}}$, the comparator output will become low. In this case, the discharge time of the $\mathrm{RC}$ network will determine the output pulse width, where the pulse width will be proportional to the amount of light sensor output voltage sampled in $\mathrm{C}_{\mathrm{S}}$, during the sampling phase. That is, the output pulse width is determined by the time that $\mathrm{V}_{\mathrm{X}}$ is higher than $\mathrm{V}_{\mathrm{R}}$. As a result, the LCD backlight can be set proportional to the ambient light level.

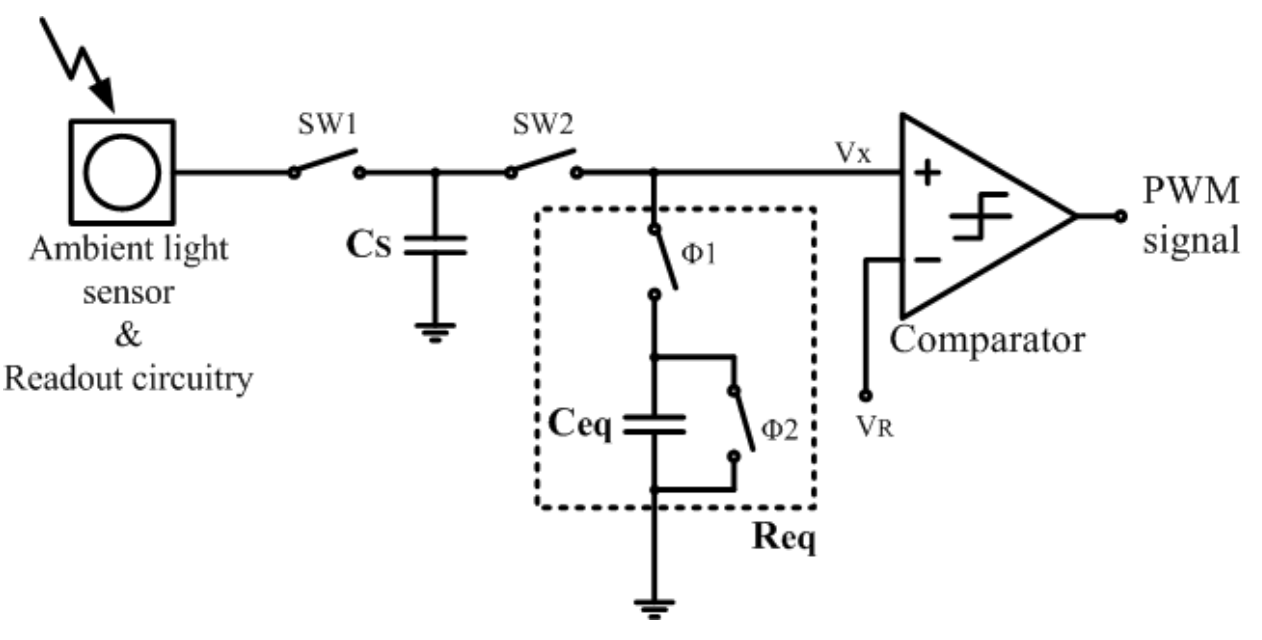

Fig. 1. Proposed switched-capacitor PWM generator architecture

Fig. 2 (a) shows simulated output waveforms of the PWM generator that corresponds to the input sampled voltage (light sensor output) from $400 \mathrm{mV}$ to $1800 \mathrm{mV}$, with step size of $100 \mathrm{mV}$. As shown, although the pulse width increment rate is slightly reduced as the pulse width increases, the pulse width is proportional to the input voltage. Fig. 2 (b) shows the output pulse duty-cycle versus the input voltage, where the dashed line is a reference for comparing the linearity. However, the relationship between discharge time to reach $V_{R}$ and the input voltage is a natural logarithm function described as

$$
t_{d i s}=\tau \cdot \ln \left(\frac{V_{X}(0)}{V_{R}}\right)
$$

where $\tau$ is time constant and $V_{\mathrm{X}}(0)$ is input voltage which is equivalent to the node voltage of $\mathrm{V}_{\mathrm{X}}$ at the beginning of the discharge phase. Even though the relationship between the output pulse width and input voltage is nonlinear, in case the required resolution of the brightness level is low, a nearly linear 
relationship can be achieved by using a limiting the operation range - for instance use the input voltage range from $400 \mathrm{mV}$ to $800 \mathrm{mV}$. Fig. 2 (c) and (d) are the measured output waveforms of the PWM generator corresponding

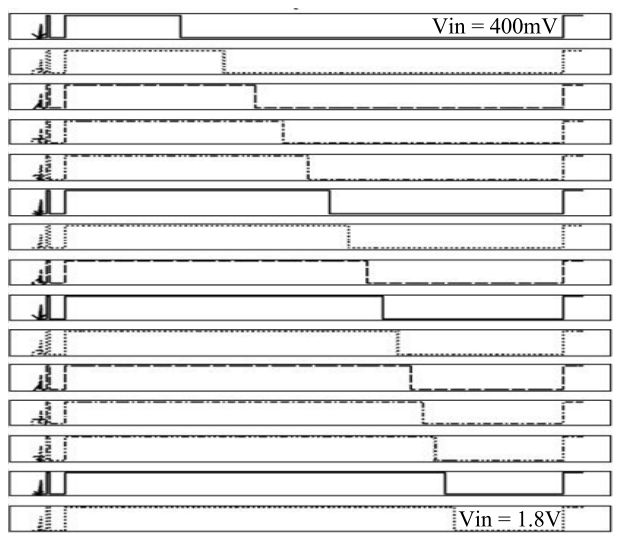

(a)

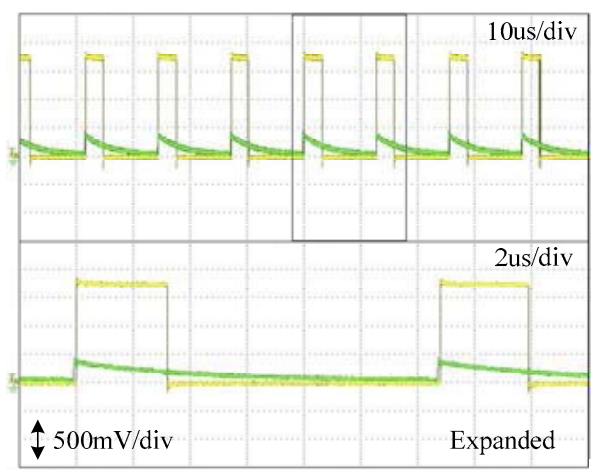

(c)

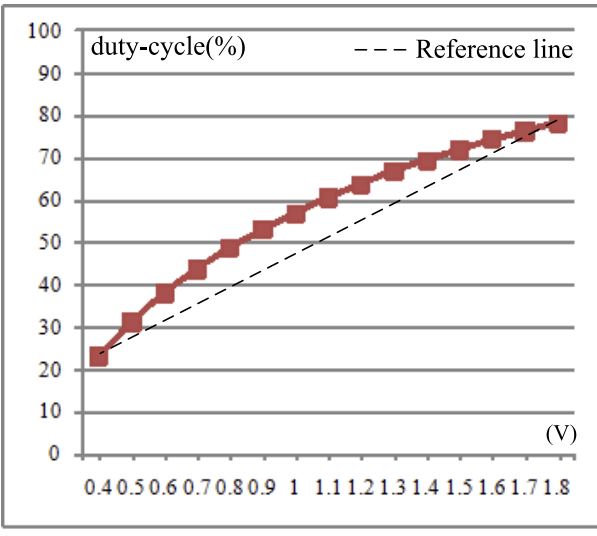

(b)

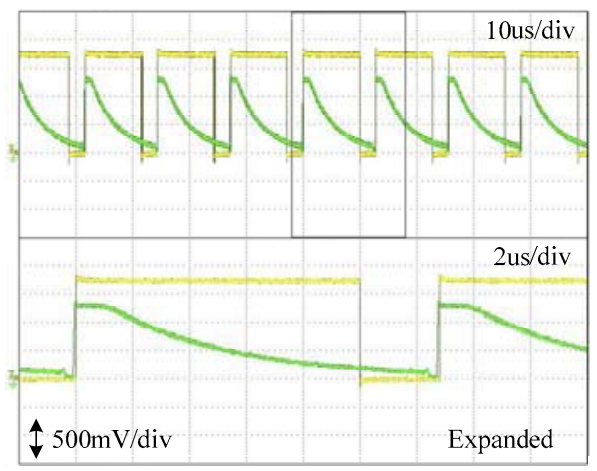

(d)

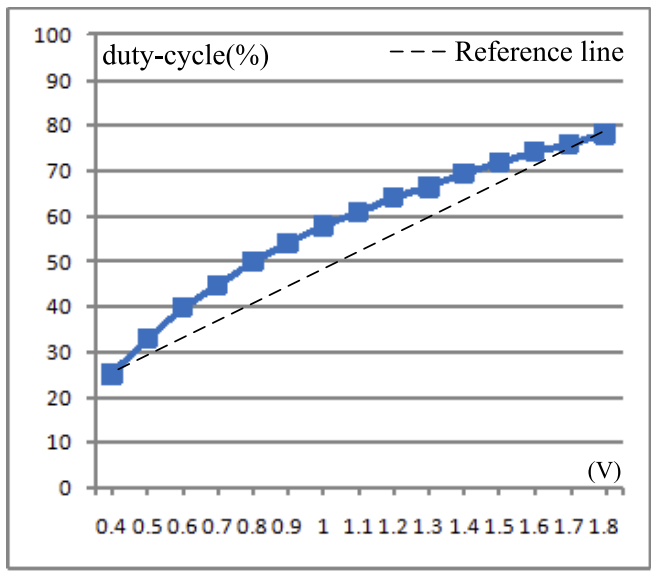

(e)

Fig. 2. (a) Simulated output waveforms for Vin $=400 \mathrm{mV}$ to $1800 \mathrm{mV}$ (b) Simulated output pulse duty-cycle versus input voltage (c) Measured output waveform for Vin $=400 \mathrm{mV}$ (d) Measured output waveform for Vin $=1800 \mathrm{mV}$ (e) Measured output pulse duty-cycle versus input voltage 
to input voltage of $400 \mathrm{mV}$ and $1800 \mathrm{mV}$, respectively.

Fig. 2 (e) shows the measured output pulse duty-cycle versus input voltage which is in accordance with the simulation results shown in Fig. 2 (b).

\section{Linearity enhancement}

The drawback of the proposed SC PWM generator is the limited linear operation range. To solve this problem, the SC resistor is replaced with a constant current source implemented with a single n-channel MOSFET, as shown in Fig. 3 (a). As a result, during the discharge phase, the charge accumulated in $\mathrm{C}_{\mathrm{S}}$ can be discharged with a constant rate. In this case, the discharge time will have a linear relationship with the input voltage that can be described as

$$
t_{d i s}=\left(\frac{C_{S}}{i_{C}}\right) \cdot\left(V_{X}(0)-V_{R}\right)
$$

where $\mathrm{i}_{\mathrm{C}}$ is the constant current flowing through the current source. Fig. 3 (b) and (c) are the simulation results using the constant current source. As shown, the output is linear through the entire input range. In addition, since the current source is only active during the discharge phase, the power consumption can be comparable to the SC PWM generator, yet more area



(a)

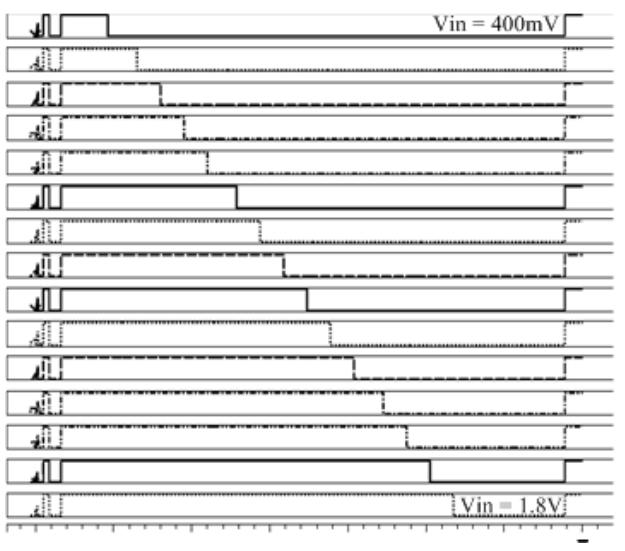

(b)

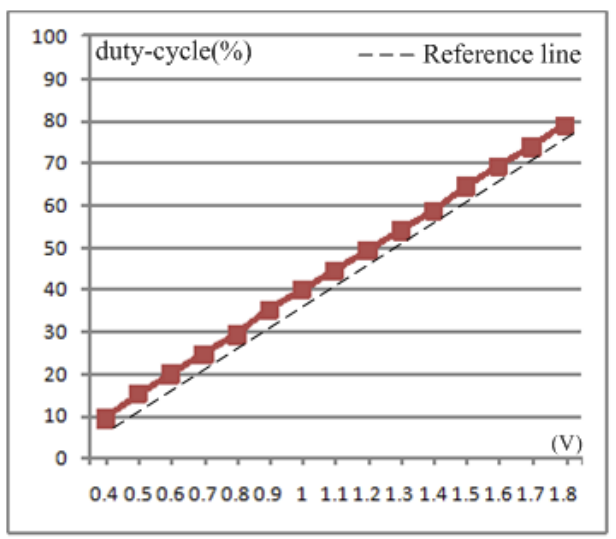

(c)

Fig. 3. (a) Proposed linearity enhancement scheme (b) Simulated output waveforms (c) Simulated output pulse duty-cycle versus input voltage 
saving with respect to the SC case is possible due to the single transistor implementation.

\section{Conclusion}

In this work, a switched-capacitor based PWM generator for on-chip LCD backlight controllers has been proposed. The proposed scheme employs a simple RC network with a comparator to generate the PWM signal corresponding to the ambient light level. Furthermore, by using a SC resistor with variable sampling clock frequency, the $\mathrm{RC}$ time constant of the backlight controller is programmable and less sensitive to process variations compared to passive resistors. The power consumption of the proposed PWM generator can be considerably reduced compared to conventional PWM generators, since it eliminates the ramp signal generator and the ADC. Although nonlinearity is an inherent problem of the RC network, a nearly linear operation can be achieved by operating the PWM generator with a limited input voltage range. However, it is also shown that the linearity of the PWM generator can be enhanced by replacing the SC resistor with a constant current source, which provides an alternative solution for higher resolution backlight controllers. Overall, due to the simple circuitry and robust operation, the proposed PWM generator is suitable for on-chip LCD backlight controllers.

\section{Acknowledgments}

This work was supported by the Sun Moon University Research Grant of 2009 and IC Design Education Center (IDEC). 